\title{
Fluorescent probes for therapeutic gas
}

\author{
Jong Seung Kim* \\ Department of Chemistry, Korea University, Seoul 02841, Korea.
}

${ }^{\star}$ Correspondence to: Dr. Jong Seung Kim, Department of Chemistry, Korea University, 145, Anam-ro, Seoul 02841, Korea. E-mail: jongskim@korea.ac.kr

How to cite this article: Kim JS. Fluorescent probes for therapeutic gas. Chem Synth 2021;1:5.

https://dx.doi.org/10.20517/cs.2021.08

Received: 15 Jul 2021 First Decision: 19 Jul 2021 Revised: 9 Aug 2021 Accepted: 9 Aug 2021 First online: 10 Aug 2021

Academic Editor: Bao-Lian Su Copy Editor: Yue-Yue Zhang Production Editor: Yue-Yue Zhang

As a newly burgeoning field, gas therapy is attracting increasing attention and the development of bioprobes for detecting therapeutic gas molecules represents a vital challenge. In the first issue of Chemical Synthesis, Prof. Qianjun He and his co-workers reviewed fluorescent probes for gas detection and their applications in gas therapy. They connected the structures and properties of the probes with their functions in support of their design, thereby helping readers in the fields of gas biomedicine, molecular imaging, synthetic chemistry, theranostics, and so on.

Fluorescence-based imaging technologies have become common tools in the life sciences ${ }^{[1]}$ and gas medicine, which represents a new approach to disease treatment, where gas molecules, such as NO, CO and $\mathrm{H}_{2}$, exhibit specific therapeutic effects for many diseases ${ }^{[2]}$. However, the therapeutic effects and biological roles of these gases highly depend on the site, concentration and duration time of the treatment ${ }^{[3]}$. Therefore, it is of significance to monitor their concentration and biodistribution in vivo, but this remains challenging, even though a large number of studies have realized targeted delivery and the controlled release of gas molecules. Thus, probes that can virtualize gas molecules are essential for studying and understanding the effects and mechanisms of gas therapy.

In the review, Gong et al. ${ }^{[4]}$ briefly introduced the structural characteristics of typical fluorophores and then summarized the gas probes based on them. In addition to their basic photophysical properties, the authors also provided information regarding their detection mechanisms. Furthermore, the advantages and disadvantages of gas detection strategies that could play a certain guiding role in the subsequent design of

The Author(s) 2021. Open Access This article is licensed under a Creative Commons Attribution 4.0 International License (https://creativecommons.org/licenses/by/4.0/), which permits unrestricted use, sharing, adaptation, distribution and reproduction in any medium or format, for any purpose, even commercially, as long as you give appropriate credit to the original author(s) and the source, provide a link to the Creative Commons license, and indicate if changes were made. 


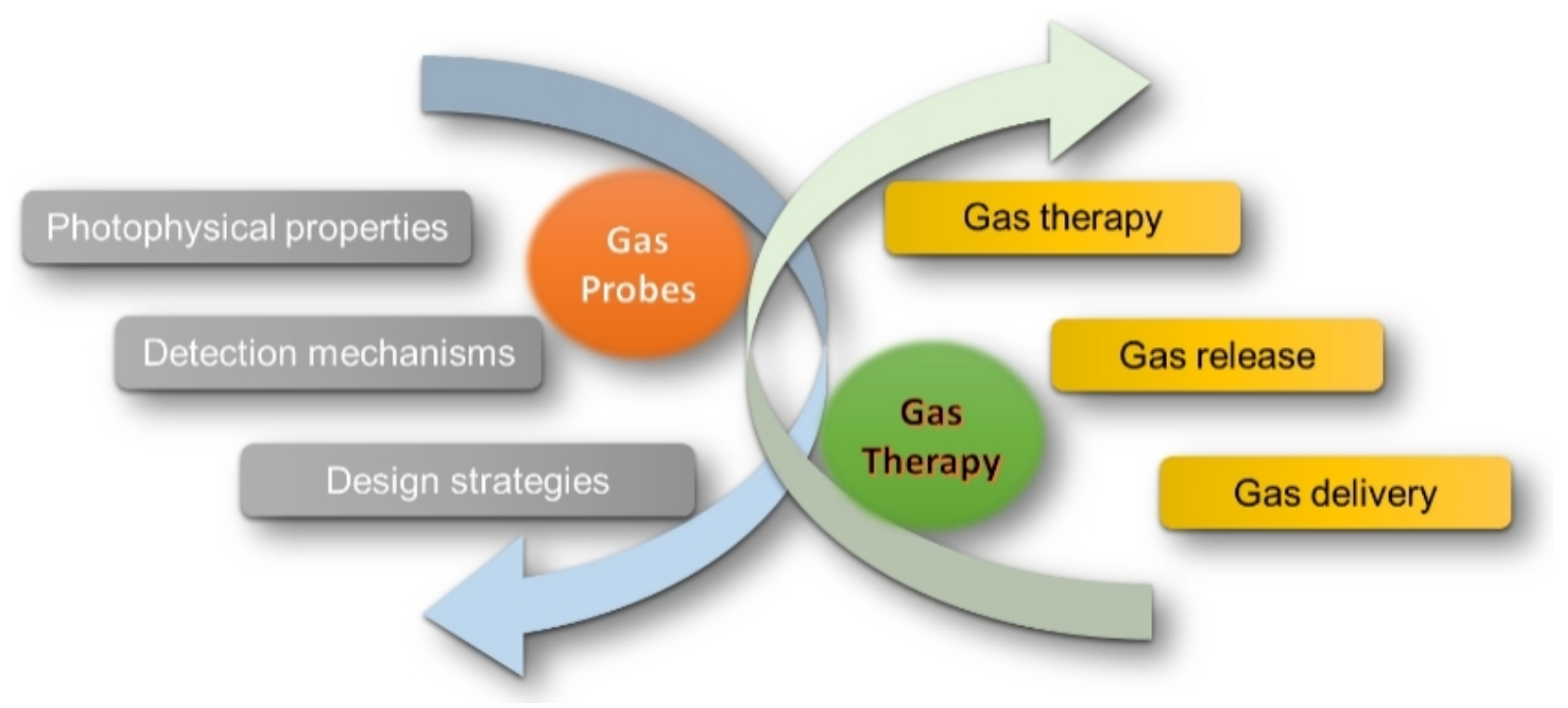

Figure 1. Gas probes and their applications in gas therapy.

advanced gas probes were also discussed [Figure 1].

Gong et al. ${ }^{[4]}$ then discussed the applications of these probes. Gas therapy was initially realized by direct inhalation ${ }^{[5]}$ or drinking gas-rich water, but to achieve a precise treatment, nanomedicines were developed ${ }^{[6]}$. The typical gas therapeutic process based on nanomedicine includes three stages: gas delivery, gas release and gas therapy [Figure 1]. In addition, with probes, the transportation and accumulation of gas carriers can be monitored, the gas release performance of nanomedicines can be evaluated and the gasinduced therapeutic effect could be determined. Therefore, with the help of probes, researchers can discover the shortcomings of nanomedicine and then make targeted improvements.

In the final section of their review, Gong et al. ${ }^{[4]}$ summarized the currently used fluorophores and imaging technologies, as well as their shortcomings in the rapid development of gas therapy. As discussed, more stable and reliable gas detection probes need to be developed and the fluorescence techniques suitable for gas therapy should not be limited to ordinary confocal or two-photon fluorescence imaging ${ }^{[7]}$. More intelligent probes $^{[8]}$ and imaging techniques ${ }^{[9]}$ are also required for a deeper understanding of the mechanisms of gas therapy.

In summary, the authors provided readers with an overview of the probes commonly used in gas therapy and their working mechanisms. Furthermore, detailed descriptions of the different roles in gas therapy were also included. This information provides invaluable insights and guidelines for the design of gas probes.

\section{DECLARATIONS}

\section{Authors' contributions}

The author contributed solely to the article.

\section{Availability of data and materials}

Not applicable. 


\section{Financial support and sponsorship}

This work was supported by the National Research Foundation of Korea (CRI project No. 2018R1A3B1052702, J.S.K.).

\section{Conflicts of interest}

The author declared that there are no conflicts of interest.

\section{Ethical approval and consent to participate}

Not applicable.

\section{Consent for publication}

Not applicable.

\section{Copyright}

(c) The Author(s) 2021.

\section{REFERENCES}

1. Power RM, Huisken J. A guide to light-sheet fluorescence microscopy for multiscale imaging. Nat Methods 2017;14:360-73. DOI PubMed

2. Chen L, Zhou SF, Su L, Song J. Gas-Mediated Cancer Bioimaging and Therapy. ACS Nano 2019;13:10887-917. DOI PubMed

3. Nakao A. Therapeutic medical gas. In: Yoshikawa T, Naito Y, editors. Gas Biology Research in Clinical Practice. Basel: KARGER; 2011. p. 15-23. DOI

4. Gong WJ, Yu ZQ, He QJ. Gas probes and their application in gas therapy. Chem Synth 2021;1:3. DOI

5. Gentile MA. Inhaled medical gases: more to breathe than oxygen. Respir Care 2011;56:1341-57; discussion 1357. DOI PubMed

6. Wang Y, Yang T, He Q. Strategies for engineering advanced nanomedicines for gas therapy of cancer. National Science Review 2020;7:1485-512. DOI

7. Pascal S, David S, Andraud C, Maury O. Near-infrared dyes for two-photon absorption in the short-wavelength infrared: strategies towards optical power limiting. Chem Soc Rev 2021;50:6613-58. DOI PubMed

8. Weinstain R, Slanina T, Kand D, Klán P. Visible-to-NIR-light activated release: from small molecules to nanomaterials. Chem Rev 2020;120:13135-272. DOI PubMed PMC

9. Verveer PJ. Advanced fluorescence microscopy: methods and protocols. New York: Humana Press; 2015. DOI 